\title{
EYA4 Promotes Cell Proliferation Through Downregulation of p27Kip1 in Glioma
}

\author{
Zhaoming Lia Ran Qiu ${ }^{\mathrm{b}} \quad$ Xia Qiuc ${ }^{\mathrm{c}}$ Tian Tian ${ }^{\mathrm{d}}$ \\ aDepartment of Oncology, the First Affiliated Hospital of Zhengzhou University, Zhengzhou, bWuhan \\ Institute of Bioengineering, Wuhan, 'Department of Medicine, Shangqiu Medical School, Shangqiu, \\ dDepartment of Neurology, the First Affiliated Hospital of Zhengzhou University, Zhengzhou, China
}

\author{
Key Words \\ Eya4 • P27Kip1 • Glioma • Cell proliferation • Prognosis
}

\begin{abstract}
Background/Aims: Accumulating evidence suggests that Eyes Absent Homologue 4 (EYA4) plays an important role in tumorigenesis and progression of various cancers. However, the role of EYA4 in glioma development is still unclear. Methods: The expression of EYA4 was examined in glioma tissues by immunohistochemistry. Cell viability and apoptosis were analyzed by CCK-8, BrdU assay, and flow cytometry. Results: We found that EYA4 was upregulated in glioma, and its expression was positively correlated with advanced tumor stage. Moreover, higher expression of EYA4 predicted a worse overall survival in patients with glioma. Forced overexpression of EYA4 enhanced glioma cell proliferation, and EYA4 suppressed the expression of p27Kip1 directly in these cells. Furthermore, Six1 was required for EYA4 to suppress the expression of p27Kip1 in glioma. Conclusion: Together, we demonstrate that EYA4 promotes cell proliferation by directly suppressing the expression of p27Kip1 in glioma.
\end{abstract}

\section{Introduction}

Gliomas are the most prevalent type of primary brain tumors in adults, accounting for more than $40 \%$ of all intracranial tumors [1,2]. According to the World Health Organization grading criteria, gliomas are histologically categorized into grades I-IV. Grade IV, also called glioblastoma multiforme (GBM), is the most malignant grade of glioma, representing $51 \%$ of all central nervous system (CNS) gliomas [3, 4]. The molecular biology of GBM is highly heterogeneous. Despite advances in the technical proficiencies of surgical and radiation oncology, patients with GBM have a poor prognosis, with a median survival of less than one year from the time of diagnosis and a five-year mortality of over 95\% [5-7]. To date, the pathogenesis of GBM is still poorly understood. Therefore, it is essential to elucidate the underlying molecular pathogenesis and to identify novel therapeutic targets for GBM.

Drosophila Eyes Absent Homologue 4 (EYA4), a component of the retinal determination gene network, functions as a transcriptional coactivator and interacts with Six1 to form a 
bipartite transcription factor essential for organ development [8, 9]. Accumulating evidence has confirmed that EYA4 plays a vital role in tumorigenesis and tumor progression of various cancers, including esophagus cancer, lung cancer and colorectal carcinoma [10-13]. However, the role of EYA4 in the development of glioma remains unknown.

Cyclin-dependent kinase inhibitor 1B (p27Kip1) is an inhibitor of cyclin-dependent kinase that in humans is encoded by the $C D K N 1 B$ gene $[14,15]$. It is involved in the regulation of the cell cycle by binding to and regulating the activity of cyclin-dependent kinases including CDK2 and CDK4 $[16,17]$. p27Kip1 has been reported to modulate cell cycle progression and chemosensitivity in human malignant glioma cells. Cytosolic p27Kip1 is closely related with cancer cell motility, survival and tumorigenicity in glioma $[18,19]$. Moreover, decreased levels of p27Kip1 are associated with a worse prognosis and shorter survival in patients with glioma [20, 21].

In the present study, we found that EYA4 was upregulated in glioma, and its levels were positively correlated with advanced tumor stage. Forced overexpression of EYA4 enhanced glioma cell proliferation, and EYA4 directly suppressed the expression of p27Kip1 in glioma cells. Furthermore, we also found that higher expression of EYA4 was associated with a worse prognosis in these patients.

\section{Materials and Methods}

\section{Cell culture}

U87MG and U251MG glioma cells (Cell bank of Chinese Academy of Sciences, Shanghai, China) were cultured in Dulbecco's modified Eagle's medium (Hyclone, Logan, UT, USA) supplemented with $10 \%$ fetal bovine serum (Hyclone, Logan, UT, USA), $0.1 \mathrm{mg} / \mathrm{ml}$ streptomycin, and $100 \mathrm{units} / \mathrm{ml}$ penicillin (Invitrogen, California, USA) in $5 \% \mathrm{CO}_{2}$ atmosphere at $37^{\circ} \mathrm{C}$.

\section{Establishment of stable cell lines}

Establishment of stable cell lines was performed as previously described. Briefly, lentiviral plasmids expressing EYA4 or p27Kip1 were co-transfected with pHelper plasmids in 293T cells. Lentiviral particles were harvested and purified with ultracentrifugation from the media after 48 hours of transfection. U87MG cells were then infected with lentiviruses encoding EYA4 or vector control. For knockdown of endogenous expression of EYA4 or Six1, lentiviral constructs expressing EYA4 shRNA (RiboBio Co., Guangzhou) or Six1 shRNA were used, respectively. The following small interfering RNA (siRNA) target sequences in the human Six1 gene (NM_005982) were selected: \#1, AGTTTGAGCTCCTGGCGTG; and \#2, TTTCTATTTACAAGTGTCC. A scrambled sequence was used as negative control for RNA interference (RNAi), which had no significant homology to any human gene sequences. Inverted and self-complementary hairpin DNA oligos targeting Six1 mRNA were obtained from Genchem Biotechnology Company (Shanghai, China). The efficiency of knockdown was evaluated by western blot analysis.

\section{Colony formation assay}

Cells $\left(2.0 \times 10^{3}\right)$ were seeded into 6-well plates in triplicate in $2 \mathrm{ml}$ of complete growth medium. The medium was changed every three days. Two weeks later, cells were stained by $0.1 \%$ crystal violet (SigmaAldrich, St. Louis, MO, USA) in methanol for $10 \mathrm{~min}$. Colonies (more than $50 \mu \mathrm{m}$ diameter) were counted directly on the plate. Statistical significance was calculated from at least three independent experiments.

\section{Cell Counting Kit-8 (CCK-8)}

Cell proliferation was evaluated using Cell Counting Kit-8 (CCK-8, Dojindo, Tokyo, Japan) according to the manufacturer's instructions. The absorbance value for each well was measured at $450 \mathrm{~nm}$ with a Multiskan FC microplate reader (Thermo scientific, Waltham, MA, USA). The experiment was conducted three independent times. 


\section{Cellular Physiology Cell Physiol Biochem 2018;49:1856-1869

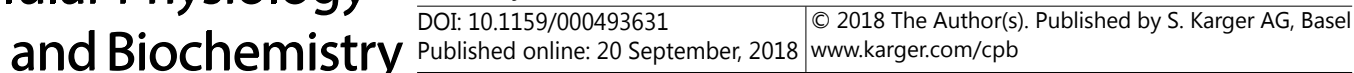

\section{BrdU incorporation}

Cells were exposed to $10 \mu \mathrm{M}$ BrdU (BD Biosciences, Mountain View, CA) for $20 \mathrm{~min}$, and fixed in 70\% ethanol. Then cells were washed with PBS and incubated with 4N HCL and 0.5\% Triton X-100 for 30 min at room temperature. Cells were neutralized with $0.1 \mathrm{M}$ sodium borate after washing with PBS, and then were labeled with FITC-conjugated BrdU antibody (BD Biosciences, Mountain View, CA) and incubated with $50 \mu \mathrm{g} / \mathrm{ml}$ propidium iodide (Sigma Chemical Company, St. Louis, MO) before being analyzed by a Becton Dickinson FACStar Plus flow cytometer.

\section{Tissue samples}

This study protocol was approved by the Research Ethics Committee of Zhengzhou University. Written informed consent was obtained from all participants. Formalin-Fixed, Paraffin-Embedded (FFPE) glioma samples were collected at the time of diagnosis from the First Affiliated Hospital of Zhengzhou University.

\section{Real-time PCR}

RNeasy mini kit (Qiagen, Germany) was used to isolate the total RNA. cDNA was prepared using the SuperScript® III First-Strand Synthesis System (Invitrogen, California, USA). To examine the mRNA abundance of p27Kip1 (CDKN1B) in U87MG cells, the following primers were used, forward primer: AACGTGCGAGTGTCTAACGG, reverse primer: CCCTCTAGGGGTTTGTG ATTCT. Quantitative PCR was performed using SYBR Green dye on an Applied Biosystems 7300 Real-time PCR system (Applied Biosystems, Foster City, CA).

\section{Western blot analysis}

Cells were lysed in cold lysis buffer as previously described [22], proteins (20-30 $\mu \mathrm{g})$ were resolved on SDS-PAGE, transferred onto PVDF membranes, and probed with antibodies for EYA4 (ab102648, Abcam, used dilution: 1:500), p27Kip1 (25614-1-AP, Proteintech, used dilution: 1:1000), Six1 (10709-1AP, Proteintech, used dilution: 1:1000) and GAPDH (sc-32233, Santa Cruz Biotechnology, used dilution: 1:2000) at $4^{\circ} \mathrm{C}$ overnight. Detection was carried out with the SuperSignal West Femto Maximum Sensitivity Substrate Trial Kit (Pierce, Rockford, IL, USA). The band images were digitally captured and quantified with a FluorChem FC2 imaging system (Alpha Innotech, San Leandro, CA, USA).

\section{Immunohistochemistry}

Immunohistochemistry (IHC) analysis was performed as previously described [23]. To verify the specificity of EYA4 antibody used in the IHC assay, we knocked down the endogenous expression of EYA4 in U87MG cells and performed xenograft tumor formation assay. The expression of EYA4 in xenograft tumor samples was then examined by IHC staining. Ki67 (ab15580, Abcam, used dilution: 1:200) and cleaved Caspase 9 (ab52298, Abcam, used dilution: 1:100) had been verified by the antibody suppliers. Immunoglobulin G (IgG) isotype controls of these corresponding antibodies used in IHC were also performed. Two scoring systems were used. (1) The protein abundance was scored semi-quantitatively based on the percentage of positive cells utilizing the following scale:,$+<25 \% ;++, 25-49 \%$;,$+++ 50-74 \%$; and,$++++ 75-100 \%$. (2) The protein abundance was assessed using a combination of the intensity and percentage of positively stained tumor cells to generate a histological score (H-score). The H-score was calculated using the following equation: $\mathrm{H}$-score $=\Sigma \mathrm{Pi}(\mathrm{i}+1)$, where $\mathrm{i}$ is the intensity score (range $0-3$ ), and $\mathrm{Pi}$ is the percentage of stained tumor cells at each intensity (range $0-100 \%$ ). This formula produced a score that ranged $100 \sim 400$, where 100 indicated that $100 \%$ of tumor cells were negative and 400 indicated that $100 \%$ of tumor cells were strongly stained. The median H-score of EYA4 was used as the cut-off to divide the study cohort into high expression and low expression groups.

\section{Luciferase assays}

Cells were transfected with expression vectors (empty vector or EYA4), the human p27Kip1 promoter pGL3-p27Kip1 and renilla reniformis luciferase (Promega, Madison, WI, USA). Cells were cultured for 24 hours and then were collected. Luciferase activities were measured using the Dual Luciferase Reporter Assay System (Promega, Madison, WI, USA) according to the manufacturer's instructions. 


\section{Cellular Physiology Cell Physiol Biochem 2018;49:1856-1869 and Biochemistry \begin{tabular}{l|l} 
DOI: 10.1159/000493631 & (c) 2018 The Author(s). Published by S. Karger AG, Basel \\
www.karger.com/cpb
\end{tabular}

\section{Chromatin Immunoprecipitation (ChIP) Assay}

The ChIP assay kit (Upstate Biotechnology, Inc., Lake Placid, NY) was used as recommended by the manufacturer. Briefly, approximately $1.0 \times 10^{7}$ cells were fixed with $1 \%$ formaldehyde for 10 min at room temperature, and washed twice with $20 \mathrm{ml}$ of cold $1 \times$ PBS, then the cells were harvested by scraping. Next, cells were lysed in $1 \mathrm{ml}$ of SDS Lysis Buffer containing Protease Inhibitor Cocktail II. Cell lysate was sonicated on wet ice four times for 15 seconds each time at intervals of 15 seconds to obtain chromatin fragments of about $200-1000 \mathrm{bp}$ nucleotides. Insoluble material was removed by centrifuged at $12,000 \times \mathrm{g}$ at $4^{\circ} \mathrm{C}$ for $10 \mathrm{~min}$. Each $100 \mu \mathrm{L}$ supernatants were diluted with $900 \mu \mathrm{L}$ of ChIP dilution buffer and preincubated with Protein $\mathrm{G}$ agarose at $4^{\circ} \mathrm{C}$ for 1 hour, and then pelleted agarose by brief centrifugation and removed 10 $\mu \mathrm{L}(1 \%)$ of the supernatant as Input. Supernatants were incubated with $5 \mu \mathrm{g}$ of EYA4 (ab32034, Abcam) and normal IgG (sc-2028, Santa cruz) overnight at $4^{\circ} \mathrm{C}$ with rotation, and then added $60 \mu \mathrm{L}$ of Protein G Agarose to each tube and incubated for 1 hour at $4^{\circ} \mathrm{C}$ with rotation. After washing using washing buffer, the immunoprecipitates were eluted and reverse cross-linked by incubation overnight at $65^{\circ} \mathrm{C}$ in elution buffer. DNA was then purified with a PCR purification kit (Qiagen, Hilden, Germany). Immunoprecipitated DNAs were analyzed by quantitative real-time PCR.

\section{Mouse xenograft assay}

Mouse xenograft assay was performed as previously reported. Briefly, the BALB/c (6-8 weeks old) athymic nude mice were purchased from Vital River Laboratory Animal Technology (Charles River Laboratories, Beijing, China). The mice were subcutaneously injected in the flank regions with $1.0 \times 10^{6}$ cells in $0.1 \mathrm{~mL}$ of PBS. The tumor size was measured every week with calipers. The tumor volume was calculated with the formula: (Length $\times \mathrm{Width}^{2}$ )/2. Four weeks after implantation, mice were euthanized by asphyxiation in $\mathrm{CO}_{2}$ chamber and tumors were excised and examined. All procedures were conducted in accordance to Animal Care and Use Committee guidelines of Zhengzhou University.

\section{Analysis of microarray data}

Oncomine Cancer Microarray database (http://www.oncomine.org) was used to study gene expression of EYA4 in glioma samples as we previously described. Gene expression data were also obtained from The Cancer Genome Atlas (TCGA) dataset and NCBI Gene Expression Omnibus (GEO) database (accession numbers: GSE4290 and GSE7696). Expression data for EYA4 were log transformed, median centered per array, and the standard deviation was normalized to one per array.

The co-expression analysis of EYA4 and p27Kip1 was performed in TCGA GBM dataset, GSE16011 and GSE4290 databases using the co-expression analysis module of the R2 microarray analysis and visualization platform (http://r2.amc.nl). The univariate survival analysis within the glioblastoma dataset of the TCGA $(\mathrm{n}=504), \mathrm{GSE} 16011(\mathrm{n}=273)$ and GSE43378 $(\mathrm{n}=50)$ was performed using the Kaplan-Meier analysis module of the R2 microarray analysis and visualization platform (http://r2.amc.nl). The cutoff values of EYA4 in TCGA, GSE16011 and GSE43378 datasets were 14.8 (207327_at), 22.7 (238877_at) and 38.2 (203706_s_at), respectively.

\section{Statistical analysis}

All experiments were performed independently three times, unless otherwise stated. Data were expressed as mean \pm standard error of the mean (SEM). Between groups and among groups comparisons were conducted with Student's t-test and ANOVA, respectively. Mann-Whitney U test was used for nonparametric variables. The association of EYA4 expression and clinical characteristics was analyzed by Chi-square or Fisher's two-tailed exact test. Statistical analysis was performed using GraphPad Prism software version 4.0 (PRISM4) (GraphPad Software Inc, LaJolla, CA), and $p<0.05$ was considered significant.

\section{Results}

EYA4 is upregulated in glioma

To investigate the role of EYA4 in glioma development, we examined the expression of EYA4 in glioma. First, EYA4 mRNA abundance was examined in glioma by querying the ONCOMINE database [24]. Three databases including TGGA, GSE7696 and GSE4290 were 
analyzed. We found that the expression of EYA4 mRNA was significantly higher in GBM than in the non-tumor brain tissues in three microarray expression studies. EYA4 mRNA expression showed an increase of 1.614-, 1.977- and 1.666- fold in TCGA, GSE7696 and GSE4290, respectively (Fig. 1A). Then the expression of EYA4 protein was examined in 88 glioma tissues and 19 non-tumor brain tissues. The results showed that EYA4 expression in tumor tissues was significantly higher than that of the non-tumor brain tissues (Fig. 1B and C). Verification of the specificity of EYA4 antibody was shown in Fig. 1B.

Next, we analyzed the correlation between the expression pattern of EYA4 and the clinicopathological characteristics in glioma (Table 1). Overexpression of EYA4 was detected in 27 of 43 patients with high-grade gliomas (grades III / IV), and the levels of EYA4 expression were significantly correlated with higher tumor stage ( $p=0.032$; Table 1$)$.

We further examined the correlation of EYA4 expression with prognosis in patients with glioma. The survival rate was analyzed using three public glioma datasets. Univariate analysis of survival was performed using Kaplan-Meier analysis module of the R2 microarray analysis and visualization platform (http://r2.amc.nl). In TCGA dataset ( $\mathrm{n}=504)$, patients with glioblastoma were divided into EYA4 high expression group (EYA4 high; $n=252$ ) and EYA4 low expression group (EYA4 low; n=252). Kaplan-Meier analysis indicated that glioblastoma patients with high expression of EYA4 had an inferior overall survival (Fig. 1D; $p=0.035)$. In the GSE16011 dataset $(n=273)$, patients with high EYA4 expression had a worse overall survival than patients with low EYA4 expression (Fig. 1D; $p=1.2 \mathrm{E}-13$ ). Similarly, in the GSE43378 dataset, high level of EYA4 predicted poor prognosis in glioma patients, even though it didn't achieve statistical significance (Fig. 1D; $p=0.057$ ).

\section{EYA4 promotes cell proliferation in glioma cells}

To address the functional role of EYA4 in glioma, we examined the effect of EYA4 on the viability of U87MG cells. We first established U87MG cells with stable expression of EYA4 using lentiviral transduction, and then assessed cell viability through CCK-8 cell growth assay and colony formation assay. The CCK-8 cell growth assay showed that the proliferation rate of U87MG and U251MG cells overexpressed with EYA4 was significantly higher than that of vector control cells $(*, p<0.05$; Fig. $2 \mathrm{~A}$ and $2 \mathrm{C}$ ). Colony formation assay of U87MG cells showed that the numbers of colonies carrying vector control and EYA4 were $45.8 \pm 4.2$ and $66.0 \pm 5.6$, respectively ( $p=0.01$; Fig. $2 \mathrm{~B}$ ). A similar colony formation result was got from U251MG cells $(p<0.01$; Fig. 2D).

To further test whether EYA4 is required for the cell viability in glioma, endogenous expression of EYA4 was knocked down by lentivirus-mediated shRNA interference in U87MG cells. The results showed that the number of EYA4-knockdown cells was significantly less than the number of shRNA control cells 4 days after plating $\left({ }^{*}, p<0.05\right.$; Fig. 2E). Suppression of EYA4 resulted in decline in the number of colonies compared to the shRNA control group revealed by colony formation assay. The numbers of colonies of shRNA control, shRNA-EYA4-1 and shRNA-EYA4-2 group were 78.9 $\pm 4.9,57.0 \pm 4.3$ and $50.1 \pm 4.4$, respectively (shRNA control vs shRNA-EYA4-1, $p=0.004$; shRNA control vs shRNA-EYA4-2, $p=0.005$; Fig. 2F). The EYA4-knockdown efficiency was determined by western blot as shown in Fig. 2G. To further validate the above findings in xenograft tumor model, U87MG cells with stable expression of EYA4 or vector control were subcutaneously injected into two groups of nude mice. Tumors derived from EYA4 group grew more rapidly than those from the vector control group as assessed by tumor volume. Four weeks later, the mean tumor volume of EYA4 group and vector control group were $(0.69 \pm 0.14) \mathrm{cm}^{3}$ and $(0.36 \pm 0.09) \mathrm{cm}^{3}$, respectively $(*, p<0.05$; Fig. $2 \mathrm{H})$. The average tumor weight of EYA4 group and vector control group were $(0.73 \pm$ $0.17) \mathrm{g}$ and $(0.34 \pm 0.07) \mathrm{g}$, respectively $(*, p<0.05$; Fig. $2 \mathrm{I})$.

We further determined the effect of EYA4 on DNA synthesis and cell apoptosis in U87MG cells. As shown in Fig. 3A, the BrdU incorporation assay showed that the number of BrdU positive cells in EYA4 group was at least 1.5-fold higher than that in vector control group ${ }^{*}$, $P<0.05$; Fig. 3A). Cell cycle analyses showed that overexpression of EYA4 leading to an increased cell proliferation index (PI) from $34 \%$ to $47 \%$ in U87MG cells $(*, P<0.05$; Fig. 3B). However, 


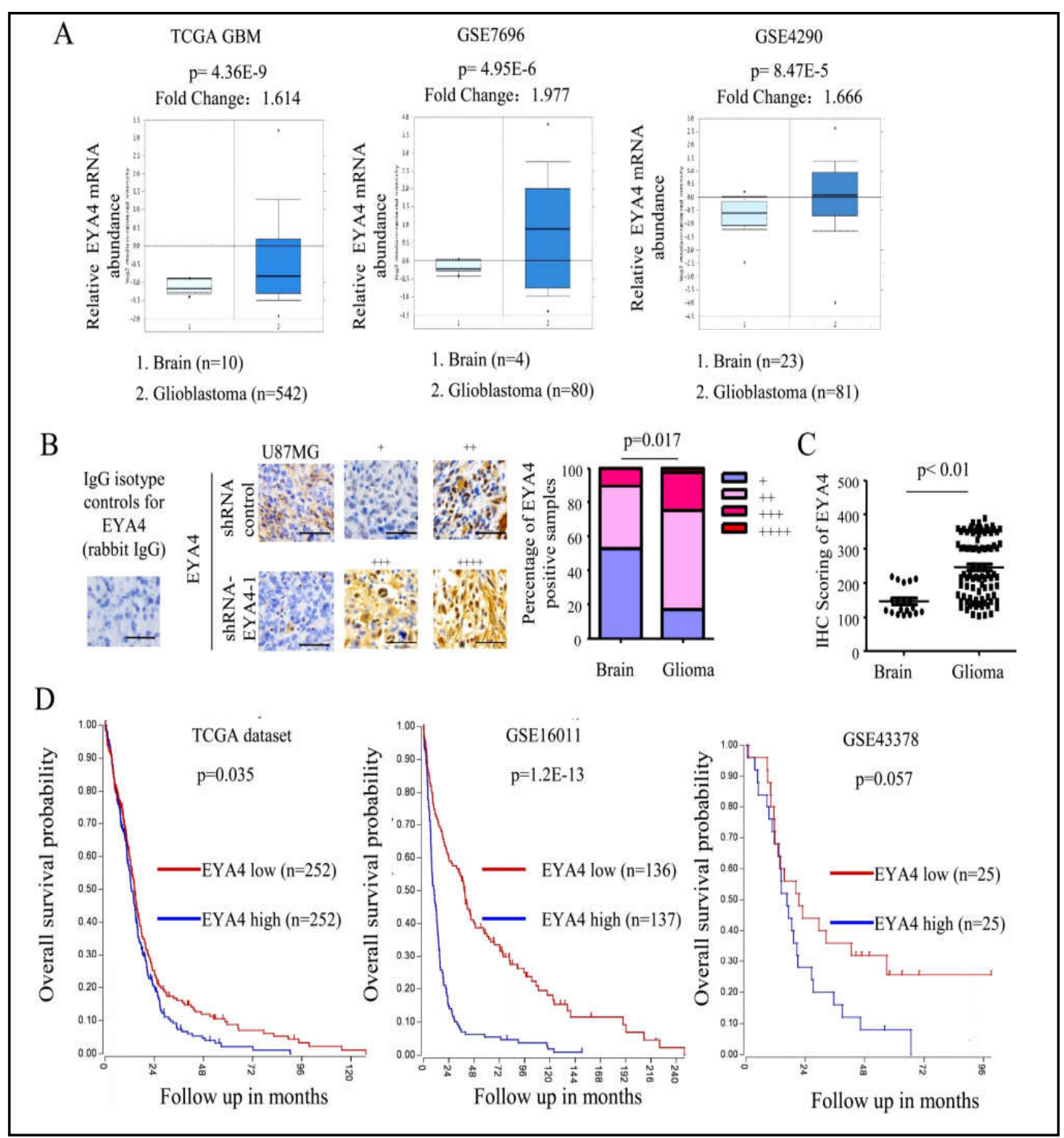

Fig. 1. EYA4 is upregulated in glioma. A. The expression of EYA4 was upregulated in glioma compared with non-tumor brain tissues. All data, including fold change and p-values, were calculated from ONCOMINE (www.oncomine.org). B and C. The protein abundance of EYA4 in 88 glioma tissues and 19 non-tumor brain tissues examined by immunohistochemisty. Verification of EYA4 antibody was shown in the left panel. Expression levels of EYA4 were scored semi-quantitatively based on the percentage of positive cells according to the following scale:,$+<25 \% ;++, 25-49 \% ;+++, 50-74 \%$; and,$++++ 75-100 \%$. Scale bars $=50$ $\mu \mathrm{m}(\mathrm{B})$, and expression levels of EYA4 were scored by H-score (C). Chi-square and Mann-Whitney U tests were used to analyze the statistical significance. D. The univariate analysis of survival was performed using the Kaplan-Meier analysis module of the R2 microarray analysis and visualization platform. Analysis of TCGA (left panel; $n=504, p=0.035$ ) and GSE16011 (middle panel; $n=273, p=1.2 \mathrm{E}-13$ ) datasets indicated that patients with high expression of EYA4 had a worse overall survival. Right panel, high level of EYA4 showed a trend to predict a worse prognosis of glioma patients in the GSE43378 dataset $(p=0.057)$. 
the apoptosis assay showed no difference between vector control and EYA4 group. (NS, not significant; Fig. 3C). In our xenograft tumor samples, the number of Ki67 positive cells was much higher in EYA4 group than in vector control group (Fig. 3D), which was in accordance with the above findings. On the contrary, the expression of cleaved Caspase 9 showed no difference between these two groups. Collectively, these results suggested that EYA4 promoted cell proliferation in glioma cells.

\section{EYA4 suppresses the expression of p27Kip1 in glioma}

The cell cycle
Table 1. The relationship between EYA4 expression and clinical characteristics of patients with glioma. Abbreviation: KPS, Karnofsky performance score

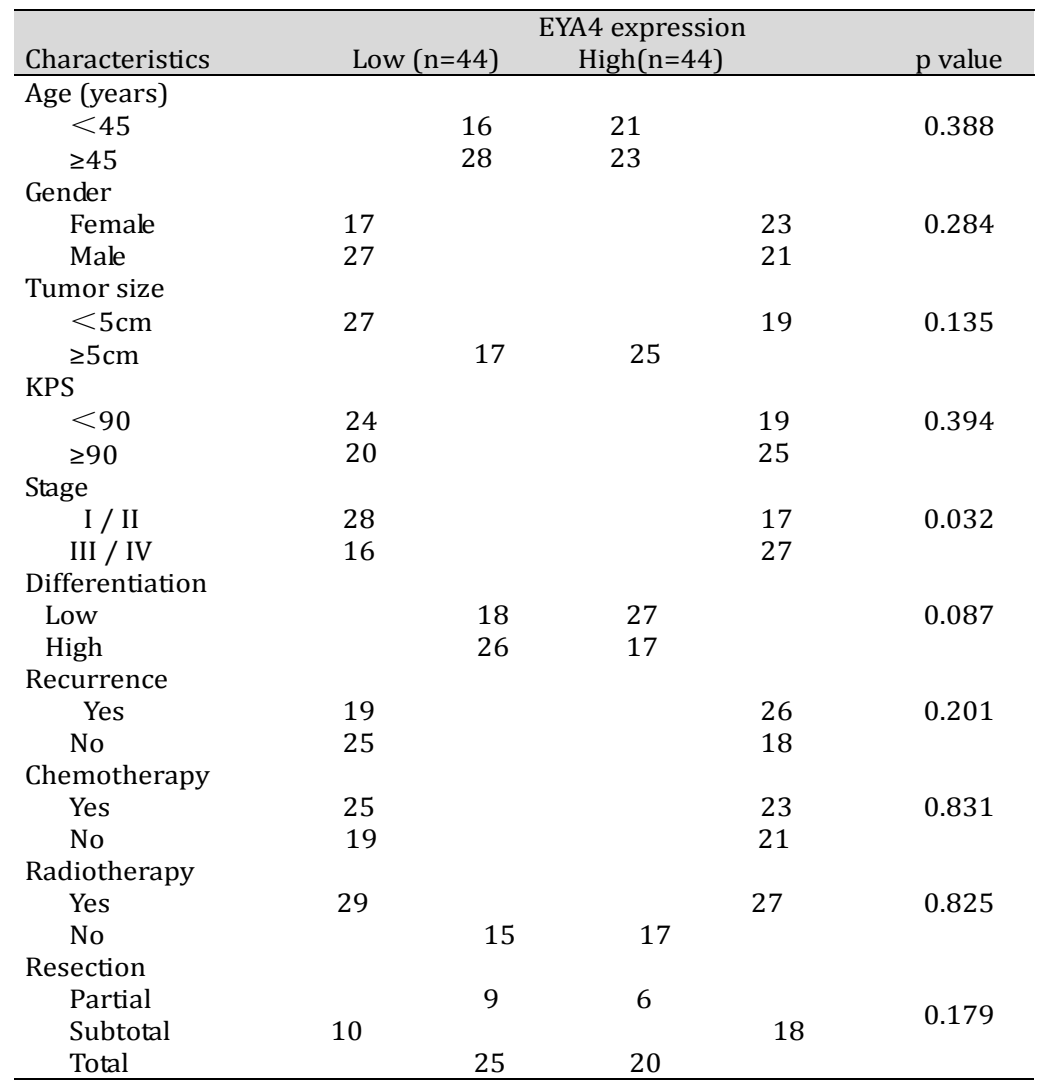

inhibitor p27Kip1 plays a crucial role in tumorigenesis and progression of glioma [25, 26]. In the present study, we found that the expression of p27Kip1 was suppressed by EYA4 in glioma cells. As shown in Fig. 4A, the expression of p27Kip1 protein was significantly lower in U87MG cells transduced with EYA4 than in vector control cells. We then examined whether EYA4 could regulate the expression of p27Kip1 at the transcription level. The quantitative PCR analysis showed that the expression of p27Kipl mRNA in cells transduced with EYA4 was decreased by at least $60 \%$ as compared to vector control cells $\left(^{*}, p<0.05\right.$, Fig. 4B). Futhermore, co-expression analysis of EYA4 and p27Kip1 in three independent glioma microarray datasets showed that EYA4 was negatively correlated with p27Kip1 in gliomas (TCGA: $\mathrm{r}$ : -0.185, $p=1.4 \mathrm{E}-5$; GSE16011: $\mathrm{r}$ : -0.311, $p=8.7 \mathrm{E}-8$; and GSE4290: $\mathrm{r}=-0.288$, $p=3.1 \mathrm{E}-4$; Fig. 4C).

Next, to explore whether EYA4 could directly regulate the promoter activity of p27Kip1 in glioma cells, U87MG cells were transfected with the human p27Kip1 promoter reporter (p27Kip1-Luc) in the presence of increasing concentrations of EYA4 plasmids. The results showed that EYA4 significantly suppressed the luciferase activity of p27Kip1 promoter reporter in a dose dependent manner $(*, p<0.05$, Fig. 4D). To determine whether EYA4 could bind to the p27Kip1 promoter in chromatin, a chromatin immunoprecipitation (ChIP) analysis was performed in U87MG cells. It has been reported that EYA protein can't directly bind to DNA, and it usually acts as a co-factor for the Six family proteins, which are transcription factors binding to DNA directly $[8,27]$. We found a putative Six1-binding site (GGGTATCA) in the promoter region of the p27Kip1 gene, at -1670 from the transcription star site. Normal IgG bound to the p27Kip1 promoter was used as negative control. As shown in Fig. 4E, EYA4 could bind to the p27Kip1 promoter between -1811 and -1632 , whereas an irrelevant sequence far from the transcriptional start site (-5000 to -5175) could not bind to 


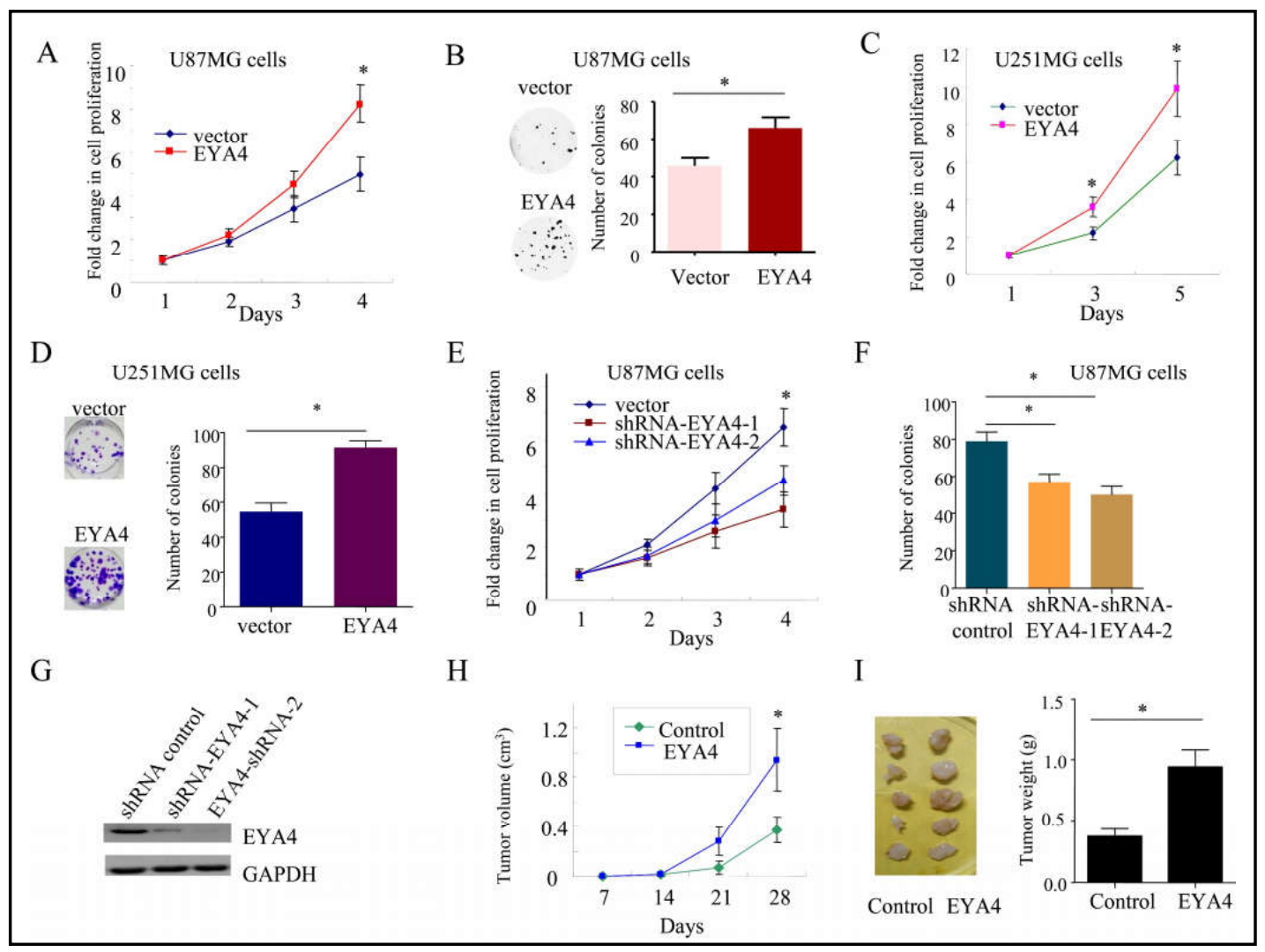

Fig. 2. EYA4 promotes cell proliferation in glioma cells. A, B. CCK-8 cell growth assay (A) and colony formation assay (B) of U87MG cells stably transfected either with EYA4 or control plasmids. Student's t-test was used to analyze the statistical significance of difference in numbers of colonies in different groups; Data were pooled from three independent experiments. $n=9$ in each group; Bars, s.e.m.; *, $p<0.05$. C, D. CCK-8 cell growth assay (C) and colony formation assay (D) of U251MG cells stably transfected either with EYA4 or control plasmids. E, F. CCK-8 cell growth assay (E) and colony formation assay (F) of U87MG cells stably transfected either with EYA4 shRNAs (shRNA-EYA4-1 and shRNA-EYA4-2) or scramble control. One-way ANOVA was used to analyze the statistical significance of difference in numbers of colonies contained in different groups; Data were pooled from three independent experiments. $n=9$ in each group; Bars, s.e.m.; *, $\mathrm{p}<0.05$. G. The EYA4-knockdown efficiency was determined by western blot. H. in vivo in xenograft tumor model. U87MG cells with stable expression of EYA4 or vector control were subcutaneously injected into two groups of nude mice. Tumor volumes were measured every week and were calculated using the formula: $\left(\right.$ Length $\times$ Width $\left.^{2}\right) / 2$. I. Representative subcutaneous tumor xenografts and the weight of tumors. Statistical significance of the tumor weight in different groups was determined using Student's t-test. $n=5$ in each group. Bars, s.e.m.; *, p<0.05.

EYA4. In summary, our data demonstrated that EYA4 suppressed the expression of p27Kip1. Therefore, p27Kip1 could be considered as an in vivo target of EYA4 in glioma cells.

\section{EYA4 enhances cell proliferation through suppression of p27Kip1 in glioma}

We next investigated whether EYA4 could promote cell proliferation through suppression of p27Kip1 in glioma cells. To this end, p27Kip1 was forced overexpressed in U87MG cells with stable expression of EYA4, and then cell growth assay and colony formation assay were performed. As a result, the cell growth assay demonstrated that overexpression of EYA4 significantly enhanced cell proliferation; however, this growth advantage conferred by EYA4 was compromised after re-expression of p27Kip1 in glioma cells $(*, p<0.05$, Fig. 5A). The numbers of colonies of vector control group, EYA4 group and EYA4+p27Kip1 group were (75.3 \pm 4.8$),(102.0 \pm 6.1)$ and $(72.7 \pm 4.3)$, respectively $(*, p<0.05$, Fig. 5B). Cell cycle and 


\section{Cellular Physiology Cell Physiol Biochem 2018;49:1856-1869 and Biochemistry \begin{tabular}{l|l} 
DOI: 10.1159/000493631 & $\mid \begin{array}{l}\text { O } 2018 \text { The Author(s). Published by S. Karger AG, Basel } \\
\text { www.karger.com/cpb }\end{array}$
\end{tabular}

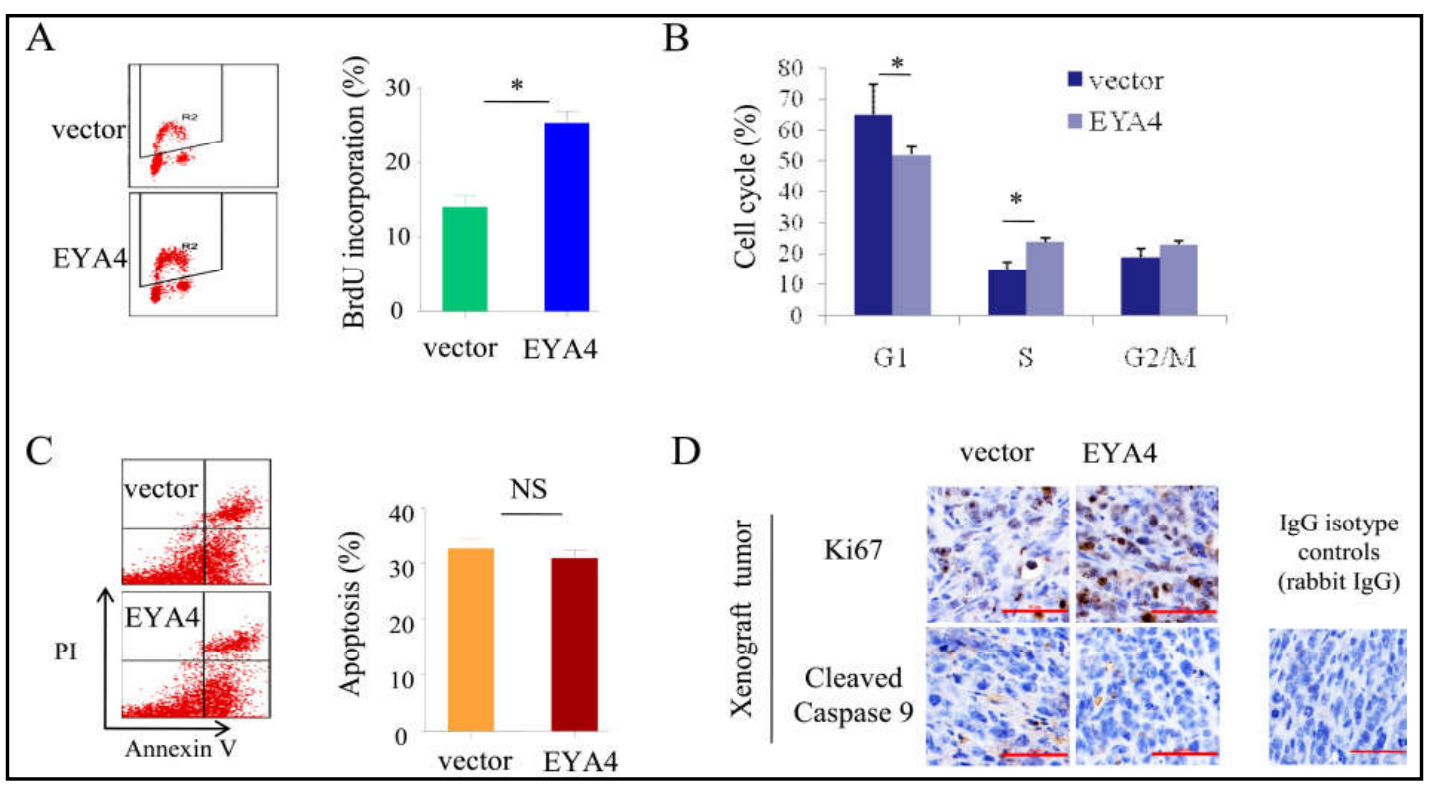

Fig. 3. The effect of EYA4 on DNA synthesis and cell apoptosis. A-C. BrdU incorporation assay (A, $n=9$ in each group), cell cycle analysis ( $B, n=9$ in each group) and apoptosis assay ( $C, n=9$ in each group) of U87MG cells stably transfected either with EYA4 or control plasmids. D. Ki67 and cleaved Caspase 9 staining in xenograft tumor samples. Statistical significance of the tumor weight in different groups was determined using Student's t-test. All experiments were performed three times independently. bars, s.e.m.; *, p<0.05. NS, not significant.

BrdU incorporation analyses also showed that overexpression of EYA4 enhanced the cell proliferation index (PI) and DNA synthesis, respectively; however, this growth advantage was compromised after re-expression of p27Kip1 ( $*, p<0.05$, Fig. $5 \mathrm{C}$ and $5 \mathrm{D})$. Taken together, these results demonstrated that suppression of p27Kip1 by EYA4 was essential for EYA4induced cell proliferation in glioma cells.

Six1 is required for EYA4 to suppress the expression of p27Kip1 in glioma

Members of the Six family have been reported to induce nuclear translocation of Eya proteins and direct them bind to specific promoter sequences. The complex exerts both activator and repressor functions [8, 9, 28-30]. Therefore, our additional aim in this study was to test whether Six1 was required for EYA4 to suppress the expression of p27Kip1. To this end, we first knocked down the endogenous Six1 expression in U87MG cells with stable EYA4 expression, and then examined the changes of p27Kip1 expression at both mRNA and protein levels. The results showed that EYA4 could suppress the expression of p27Kip1 mRNA and protein, however, the suppression effect of EYA4 on p27Kip1 expression was abolished after knockdown of endogenous Six1 in glioma cells $(*, p<0.05$, Fig. 6A and 6B). Similarly, luciferase assay showed that Six1 was required for transcriptional inactivation of p27Kip1 by EYA4 $(*, p<0.05$, Fig. 6C). Moreover, our study found that knockdown of Six1 dramatically decreased the binding activity of EYA4 to p27Kip1 promoter as determined by ChIP assay $(*, p<0.05$, Fig. 6 D). Therefore it was inferred that Six1 was required for EYA4 to suppress the expression of p27Kip1 in glioma.

\section{Discussion}

The Eyes absent (EYA) gene family which has been identified as a key regulator for eye development in Drosophila is now implicated in processes as disparate as organ development, DNA damage repair, tumorigenesis, angiogenesis and cancer metastasis [31, 32]. EYA4, as 


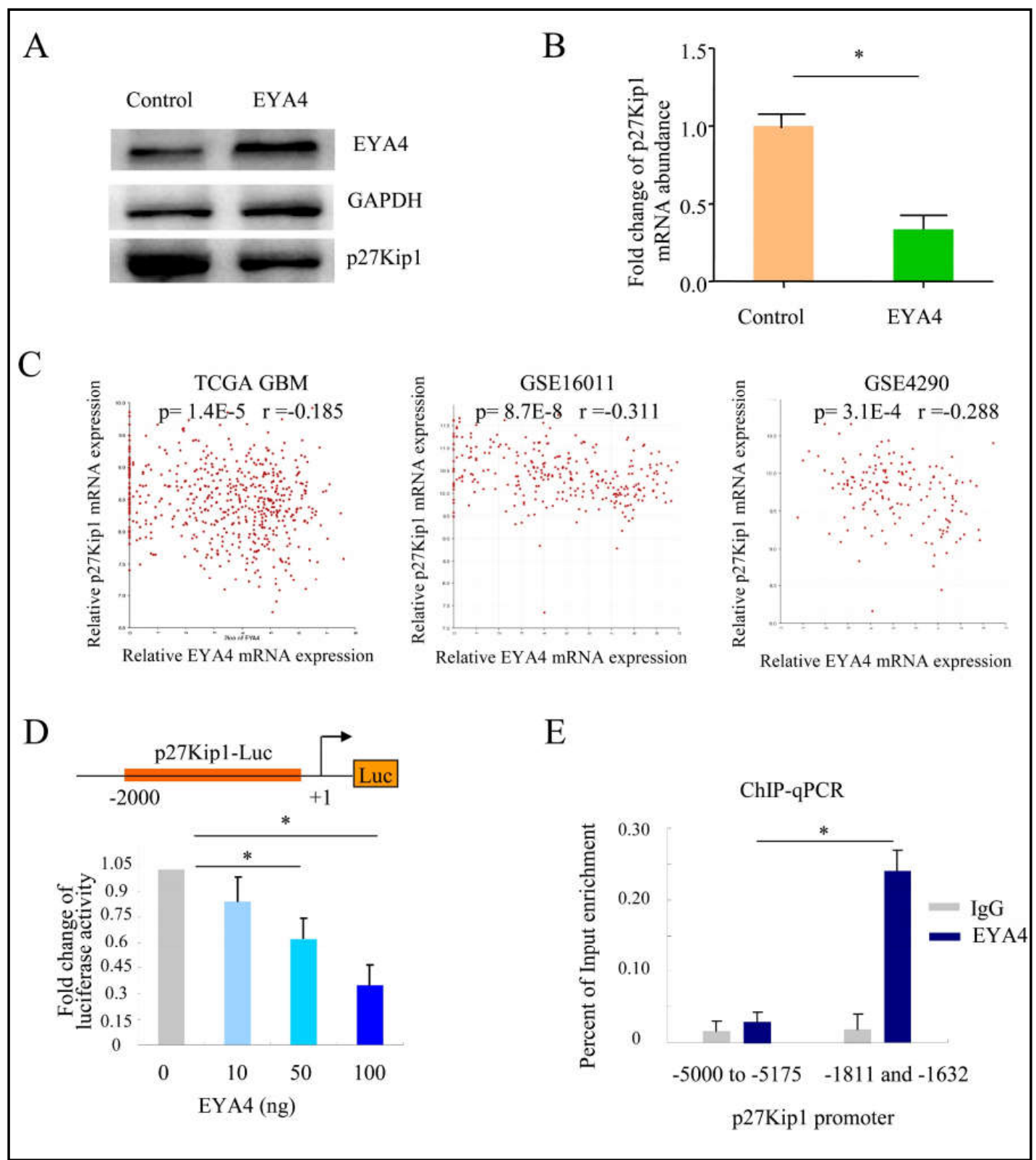

Fig. 4. EYA4 suppresses the expression of p27Kip1 in glioma. $A, B$. The protein $(A)$ and mRNA (B, $n=9$ in each group) changes of p27Kip1 affected by EYA4 in U87MG cells. C. Co-expression analysis of EYA4 and p27Kip1 in three independent glioma microarray datasets using the gene correlation module of the R2 microarray analysis and visualization platform. D. EYA4 directly regulated the promoter activity of p27Kip1 in glioma cells. U87MG cells were transfected with the human p27Kip1 promoter reporter (p27Kip1-Luc) in the presence of increasing concentrations of EYA4 plasmids, and luciferase activities were measured using the Dual Luciferase Reporter Assay System (Promega, Madison, WI, USA). $n=9$ in each group. E. ChIP analysis indicated that EYA4 could bind to the p27Kip1 promoter between -1811 and -1632, whereas an irrelevant sequence far from the transcriptional start site did not bind to EYA4. Statistical significance between two groups was determined using Student's t-test. All experiments were performed three times independently. Bars, s.e.m.; *, p<0.05. 


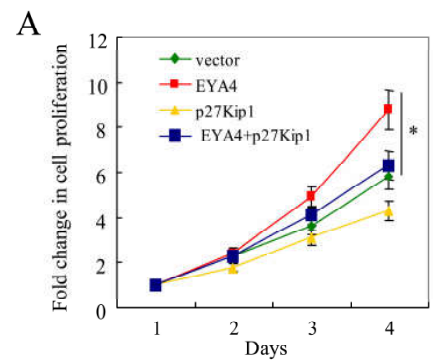

C

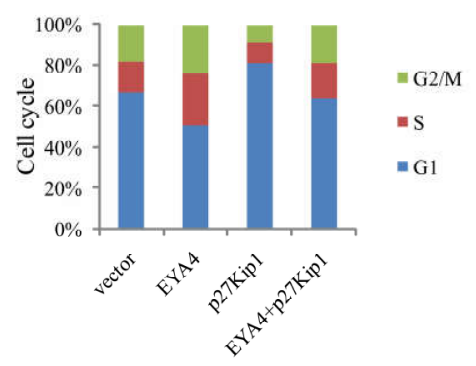

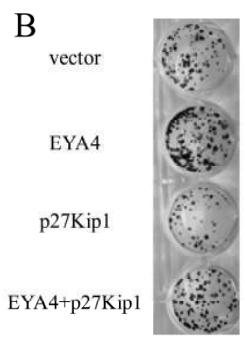

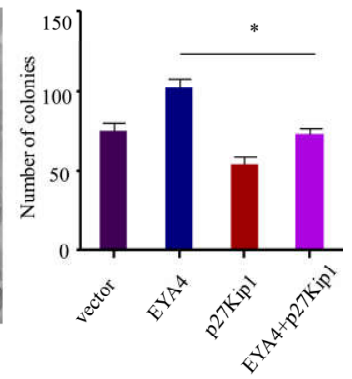

D

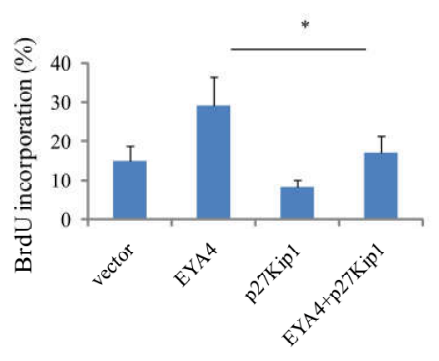

Fig. 5. EYA4 enhances cell proliferation through suppression of p27Kip1 in glioma. CCK-8 cell growth assay (A), colony formation assay (B), cell cycle analysis (C) and BrdU incorporation assay (D) showed that suppression of p27Kip1 was essential for EYA4-induced cell proliferation of glioma cells. Statistical significance between two groups was determined using Student's t-test. All experiments were performed three times independently. Bars, s.e.m. ; ${ }^{*}, \mathrm{p}<0.05$.

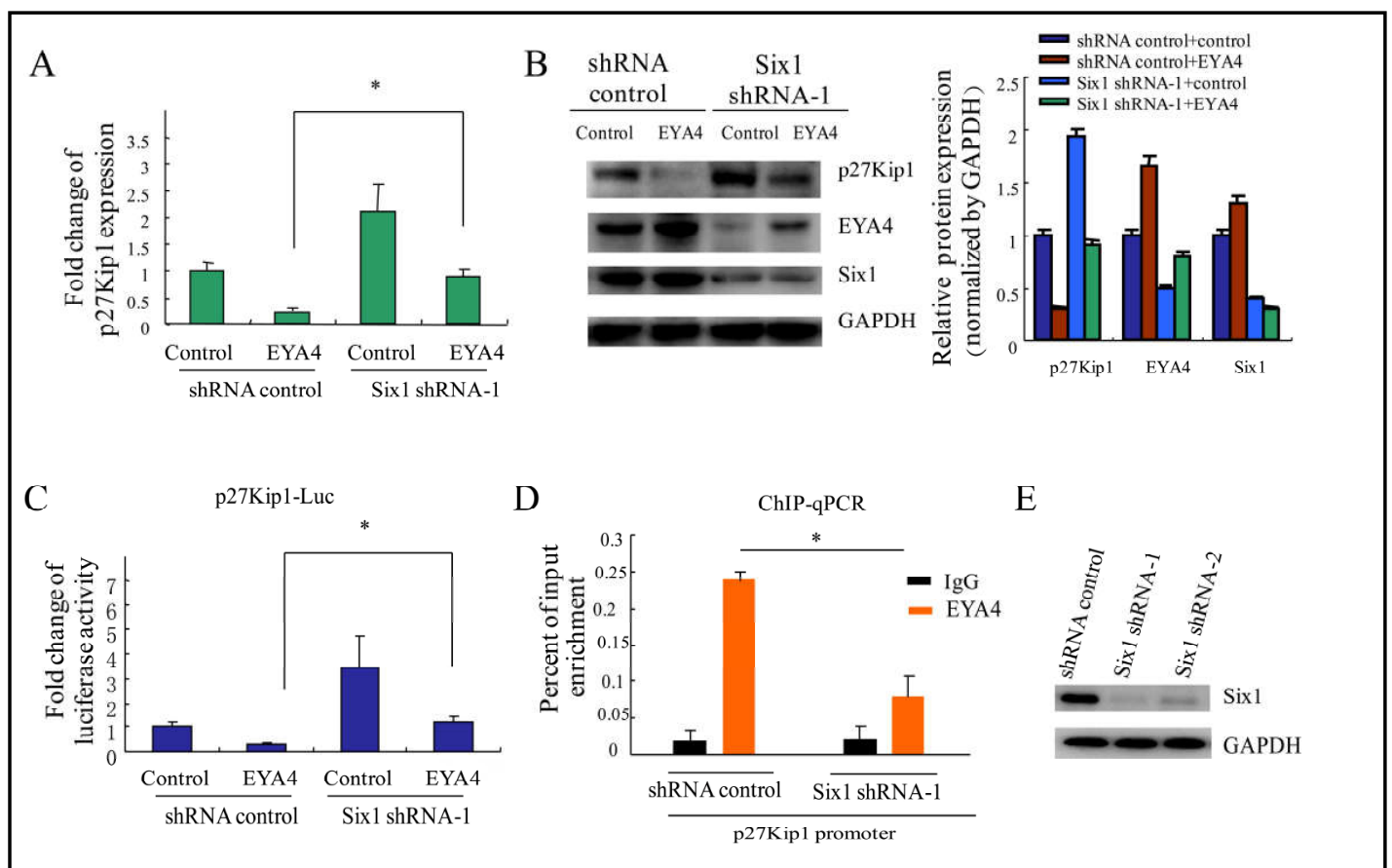

Fig. 6. Six1 is required for EYA4 to suppress the expression of p27Kip1 in glioma. A, B. The changes in mRNA (A) and protein (B) levels of p27Kip1 after inhibition of endogenous Six1 expression in U87MG cells. C. Luciferase assay showed that Six1 was required for transcriptional inactivation of p27Kip1 by EYA4. D. ChIP assay demonstrated that knockdown of Six1 dramatically decreased the activity of EYA4 on binding to p27Kip1 promoter. Statistical significance between two groups was determined using Student's t-test. All experiments were performed three times independently. Bars, s.e.m.; *, p<0.05. 


\section{Cellular Physiology Cell Physiol Biochem 2018;49:1856-1869 and Biochemistry \begin{tabular}{c|c|c|} 
DOI: 10.1159/000493631 & $\begin{array}{l}\text { O } 2018 \text { The Author(s). Published by S. Karger AG, Basel } \\
\text { www.karger.com/cpb }\end{array}$
\end{tabular}

one of the EYA family members, has been reported to play an important role in various cancers $[11,12,33$, 34]. In this study, we found that EYA4 was upregulated in glioma and its levels were positively correlated with advanced tumor stage, which was consistent with its previously reported roles in other cancers.

The cyclin kinase inhibitor p27Kip1 is considered as a tumor suppressor that plays a critical role in cell proliferation, differentiation, and apoptosis [35-

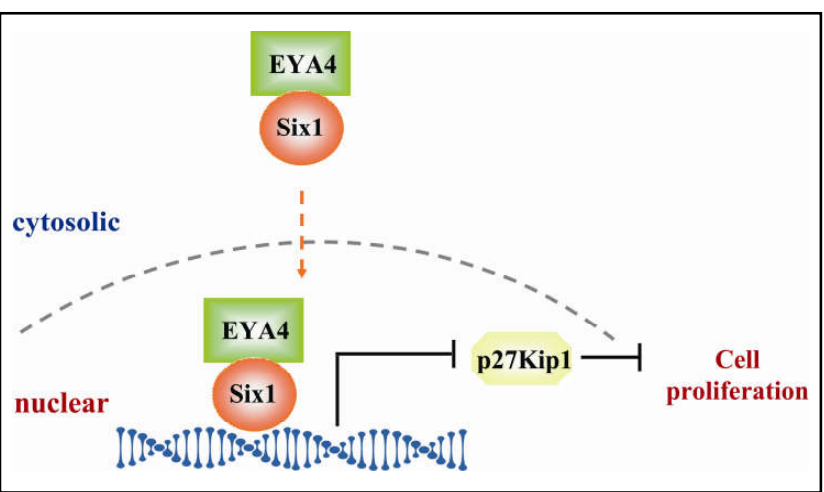

Fig. 7. Schematic figure to illustrate the new signaling pathway of EYA4/Six1/p27Kip1. 38]. Dysregulation of p27Kip1 gene expression at the mRNA or the protein level was reported in various cancers [39, 40]. Especially, p27Kip1 has been implicated in tumorigenesis and oncogenic progression of gliomas $[41,42]$. Here we provide evidence that p27Kip1 is a transcriptional target of EYA4, and its expression can be suppressed by EYA4 in glioma cells. Therefore, our study demonstrates that EYA4 promotes cell proliferation through downregulation of p27Kip1 in glioma cells, further emphasizing the critical role of p27Kip1 in the development of gliomas.

Studies have shown that EYA4 interacts with Six1 to form a bipartite transcription factor essential for the regulation of various downstream targets expression [34, 43, 44]. The transcription co-activator EYA4 could be recruited in the context of local chromatin; however, it lacks intrinsic DNA-binding activity. EYA domain (ED), a large, highly conserved C-terminal domain of EYA family proteins, is essential for its ability to bind to Six and DACH proteins [31]. Our data suggest that Six 1 is required for the ability of EYA4 to suppress the expression of p27Kip1 in glioma cells (Fig. 7), which is in line with previous reports that Eya4/Six1 regulates normal cardiac function through regulation of p27Kip1 [43].

\section{Conclusion}

In conclusion, this study demonstrates that EYA4 is overexpressed in glioma, and its overexpression leads to increased glioma cell proliferation through downregulation of p27Kip1, implying the important role of EYA4 in the development of glioma.

\section{Acknowledgements}

This study was supported by funds from the National Natural Science Foundation of Henan (2014-81402380).

\section{Disclosure Statement}

The authors have no conflicts of interest to declare.

\section{References}

1 Wen PY, Reardon DA: Neuro-oncology in 2015: Progress in glioma diagnosis, classification and treatment. Nat Rev Neurol 2016;12:69-70. 


\section{Cellular Physiology Cell Physiol Biochem 2018;49:1856-1869 and Biochemistry \begin{tabular}{l|l} 
DOI: 10.1159/000493631 \\
Published 2018 The Author(s). Published by S. Karger AG, Basel
\end{tabular}

2 Reardon DA, Wen PY: Glioma in 2014: unravelling tumour heterogeneity-implications for therapy. Nat Rev Clin Oncol 2015;12:69-70.

-3 Adamson DC, Rasheed BA, McLendon RE, Bigner DD: Central nervous system. Cancer Biomark 2010;9:193210.

4 Verhaak RG: Moving the needle: Optimizing classification for glioma. Sci Transl Med 2016;8:350fs314.

5 Lenting K, Verhaak R, Ter Laan M, Wesseling P, Leenders W: Glioma: experimental models and reality. Acta Neuropathol 2017;133:263-282.

-6 Eckert M, Klumpp L, Huber SM: Cellular Effects of the Antiepileptic Drug Valproic Acid in Glioblastoma. Cell Physiol Biochem 2017;44:1591-1605.

-7 Zhao W, Peng F, Shu M, Liu H, Hou X, Wang X, Ye J, Zhao B, Wang K, Zhong C, Xue L, Gao M, Liu Y, Zhao S: Isogambogenic Acid Inhibits the Growth of Glioma Through Activation of the AMPK-mTOR Pathway. Cell Physiol Biochem 2017;44:1381-1395.

-8 Li X, Oghi KA, Zhang J, Krones A, Bush KT, Glass CK, Nigam SK, Aggarwal AK, Maas R, Rose DW, Rosenfeld MG: Eya protein phosphatase activity regulates Six1-Dach-Eya transcriptional effects in mammalian organogenesis. Nature 2003;426:247-254.

\$9 Tadjuidje E, Hegde RS: The Eyes Absent proteins in development and disease. Cell Mol Life Sci 2013;70:1897-1913.

10 Emmett RA, Davidson KL, Gould NJ, Arasaradnam RP: DNA methylation patterns in ulcerative colitisassociated cancer: a systematic review. Epigenomics 2017;9:1029-1042.

11 Hao XY, Cai JP, Liu X, Chen W, Hou X, Chen D, Lai JM, Liang LJ, Yin XY: EYA4 gene functions as a prognostic marker and inhibits the growth of intrahepatic cholangiocarcinoma. Chin J Cancer 2016;35:70.

12 Mo SJ, Liu X, Hao XY, Chen W, Zhang KS, Cai JP, Lai JM, Liang LJ, Yin XY: EYA4 functions as tumor suppressor gene and prognostic marker in pancreatic ductal adenocarcinoma through beta-catenin/ID2 pathway. Cancer Lett 2016;380:403-412.

13 Wilson IM, Vucic EA, Enfield KS, Thu KL, Zhang YA, Chari R, Lockwood WW, Radulovich N, Starczynowski DT, Banath JP, Zhang M, Pusic A, Fuller M, Lonergan KM, Rowbotham D, Yee J, English JC, Buys TP, Selamat SA, Laird-Offringa IA et al.: EYA4 is inactivated biallelically at a high frequency in sporadic lung cancer and is associated with familial lung cancer risk. Oncogene 2014;33:4464-4473.

14 Sharma SS, Pledger WJ: The non-canonical functions of p27(Kip1) in normal and tumor biology. Cell Cycle 2016;15:1189-1201.

15 Wander SA, Zhao D, Slingerland JM: p27: a barometer of signaling deregulation and potential predictor of response to targeted therapies. Clin Cancer Res 2011;17:12-18.

16 Hnit SS, Xie C, Yao M, Holst J, Bensoussan A, De Souza P, Li Z, Dong Q: p27(Kip1) signaling: Transcriptional and post-translational regulation. Int J Biochem Cell Biol 2015;68:9-14.

17 Xiea Y, Wangb R: Pttg1 Promotes Growth of Breast Cancer through P27 Nuclear Exclusion. Cell Physiol Biochem 2016;38:393-400.

18 Bhatia B, Malik A, Fernandez LA, Kenney AM: p27(Kip1), a double-edged sword in Shh-mediated medulloblastoma: Tumor accelerator and suppressor. Cell Cycle 2010;9:4307-4314.

19 Wu FY, Wang SE, Sanders ME, Shin I, Rojo F, Baselga J, Arteaga CL: Reduction of cytosolic p27(Kip1) inhibits cancer cell motility, survival, and tumorigenicity. Cancer Res 2006;66:2162-2172.

-20 He SM, Zhao ZW, Wang Y, Zhao JP, Wang L, Hou F, Gao GD: Potential role of Jun activation domain-binding protein 1 and phosphorylated p27 expression in prognosis of glioma. Brain Tumor Pathol 2012;29:3-9.

-21 Shen A, Wang Y, Zhao Y, Zou L, Sun L, Cheng C: Expression of CRM1 in human gliomas and its significance in p27 expression and clinical prognosis. Neurosurgery 2009;65:153-159.

22 Zhang Z, Luo Z, Min W, Zhang L, Wu Y, Hu X: An anti-cancer WxxxE-containing azurin polypeptide inhibits Rac1-dependent STAT3 and ERK/GSK-3beta signaling in breast cancer cells. Oncotarget 2017;8:4309143103.

23 Li Z, Lu L, Zhou Z, Xue W, Wang Y, Jin M, Qiu Y, Sun W, Fu X, Zhang X, Chang Y, Nan F, Yan J, Wang G, Sun Z, Fu X, Li L, Li X, Wang X, Wu J et al.: Recurrent mutations in epigenetic modifiers and the PI3K/AKT/mTOR pathway in subcutaneous panniculitis-like T-cell lymphoma. Br J Haematol 2018;181:406-410.

24 Rhodes DR, Yu J, Shanker K, Deshpande N, Varambally R, Ghosh D, Barrette T, Pandey A, Chinnaiyan AM: ONCOMINE: a cancer microarray database and integrated data-mining platform. Neoplasia 2004;6:1-6.

-25 Naumann U, Weit S, Rieger L, Meyermann R, Weller M: p27 modulates cell cycle progression and chemosensitivity in human malignant glioma. Biochem Biophys Res Commun 1999;261:890-896. 


\section{Cellular Physiology Cell Physiol Biochem 2018;49:1856-1869 and Biochemistry \begin{tabular}{l|l} 
DOI: 10.1159/000493631 \\
Published
\end{tabular}

26 Komata T, Kanzawa T, Takeuchi H, Germano IM, Schreiber M, Kondo Y, Kondo S: Antitumour effect of cyclindependent kinase inhibitors (p16(INK4A), p18(INK4C), p19(INK4D), p21(WAF1/CIP1) and p27(KIP1)) on malignant glioma cells. Br J Cancer 2003;88:1277-1280.

27 Blevins MA, Towers CG, Patrick AN, Zhao R, Ford HL: The SIX1-EYA transcriptional complex as a therapeutic target in cancer. Expert Opin Ther Targets 2015;19:213-225.

-28 Xu PX: The EYA-SO/SIX complex in development and disease. Pediatr Nephrol 2013;28:843-854.

29 Lopez-Rios J, Tessmar K, Loosli F, Wittbrodt J, Bovolenta P: Six3 and Six6 activity is modulated by members of the groucho family. Development 2003;130:185-195.

30 Kobayashi M, Nishikawa K, Suzuki T, Yamamoto M: The homeobox protein Six3 interacts with the Groucho corepressor and acts as a transcriptional repressor in eye and forebrain formation. Dev Biol 2001;232:315326.

-31 Liu Y, Han N, Zhou S, Zhou R, Yuan X, Xu H, Zhang C, Yin T, Wu K: The DACH/EYA/SIX gene network and its role in tumor initiation and progression. Int J Cancer 2016;138:1067-1075.

32 Popov VM, Wu K, Zhou J, Powell MJ, Mardon G, Wang C, Pestell RG: The Dachshund gene in development and hormone-responsive tumorigenesis. Trends Endocrinol Metab 2010;21:41-49.

33 Hou X, Peng JX, Hao XY, Cai JP, Liang LJ, Zhai JM, Zhang KS, Lai JM, Yin XY: DNA methylation profiling identifies EYA4 gene as a prognostic molecular marker in hepatocellular carcinoma. Ann Surg Oncol 2014;21:3891-3899.

-34 Miller SJ, Lan ZD, Hardiman A, Wu J, Kordich JJ, Patmore DM, Hegde RS, Cripe TP, Cancelas JA, Collins MH, Ratner N: Inhibition of Eyes Absent Homolog 4 expression induces malignant peripheral nerve sheath tumor necrosis. Oncogene 2010;29:368-379.

-35 Kopsiaftis S, Sullivan KL, Garg I, Taylor JA, 3rd, Claffey KP: AMPKalpha2 Regulates Bladder Cancer Growth through SKP2-Mediated Degradation of p27. Mol Cancer Res 2016;14:1182-1194.

-36 Hydbring P, Castell A, Larsson LG: MYC Modulation around the CDK2/p27/SKP2 Axis. Genes (Basel) 2017;8.

37 Baldassarre G, Belletti B: Meet me in the cytoplasm: A role for p27(Kip1) in the control of H-Ras. Small GTPases 2016;7:71-75.

-38 Serres MP, Kossatz U, Chi Y, Roberts JM, Malek NP, Besson A: p27(Kip1) controls cytokinesis via the regulation of citron kinase activation. J Clin Invest 2012;122:844-858.

-39 Ronen S, Abbott DW, Kravtsov 0, Abdelkader A, Xu Y, Banerjee A, Iczkowski KA: PTEN loss and p27 loss differ among morphologic patterns of prostate cancer, including cribriform. Hum Pathol 2017;65:85-91.

-40 Ciesielska U, Zatonski T, Nowinska K, Ratajczak-Wielgomas K, Grzegrzolka J, Piotrowska A, Olbromski M, Pula B, Podhorska-Okolow M, Dziegiel P: Expression of Cell Cycle-related Proteins p16, p27 and Ki-67 Proliferating Marker in Laryngeal Squamous Cell Carcinomas and in Laryngeal Papillomas. Anticancer Res 2017;37:2407-2415.

41 Mukherjee J, Ohba S, See WL, Phillips JJ, Molinaro AM, Pieper RO: PKM2 uses control of HuR localization to regulate p27 and cell cycle progression in human glioblastoma cells. Int J Cancer 2016;139:99-111.

-42 Galardi S, Petretich M, Pinna G, D’Amico S, Loreni F, Michienzi A, Groisman I, Ciafre SA: CPEB1 restrains proliferation of Glioblastoma cells through the regulation of p27(Kip1) mRNA translation. Sci Rep 2016;6:25219.

43 Williams T, Hundertmark M, Nordbeck P, Voll S, Arias-Loza PA, Oppelt D, Muhlfelder M, Schraut S, Elsner I, Czolbe M, Seidlmayer L, Heinze B, Hahner S, Heinze K, Schonberger J, Jakob P, Ritter O: Eya4 Induces Hypertrophy via Regulation of p27kip1. Circ Cardiovasc Genet 2015;8:752-764.

44 Zou D, Silvius D, Rodrigo-Blomqvist S, Enerback S, Xu PX: Eya1 regulates the growth of otic epithelium and interacts with Pax2 during the development of all sensory areas in the inner ear. Dev Biol 2006;298:430441. 